\title{
Microscopy of the Deformation of Tantalum
}

\author{
Matthew T. Janish ${ }^{1}$, Bill Mook ${ }^{2}$, Shraddha J. Vachhani ${ }^{3}$, Ellen Cerreta ${ }^{3}$, C. Barry Carter ${ }^{1,4}$ \\ ${ }^{1}$ Dept of Materials Science \& Engineering, U. of Connecticut, Storrs, CT \\ ${ }^{2}$ Center for Integrated Nanotechnologies (CINT), Sandia National Laboratory, Albuquerque, NM \\ ${ }^{3}$ Materials Science and Technology, Los Alamos National Laboratory, Los Alamos, NM \\ ${ }^{4}$ Dept of Chemical \& Biomolecular Engineering, U. of Connecticut, Storrs, CT
}

The deformation behavior of metals with the fcc structure is well-characterized and understood, but the same cannot be said of the bcc metals. This rather wide knowledge gap is attributable to the difference in structure and bond character of the two classes of materials. Plastic deformation in metals is achieved by the motion of dislocations, and the motion of dislocations in close-packed structures is relatively easy to predict. Because the bcc structure is not close packed (due to more complicated, partially covalent bonding), it stands to reason that dislocation motion, and therefore plastic deformation, is still not wellunderstood for the metals with this structure.

Tantalum, a refractory bcc transition metal, is used in microelectronics for capacitors and resistors, in medical applications for surgical instruments or as an implant material, in X-ray lithography for masks, and in high-temperature structural applications, such as heat exchangers. While the bulk metal has the bcc structure, deposited thin films can be amorphous or have the beta-Ta or fcc structure [1, 2]. The fcc phase and small regions of a hcp phase have been identified in heavily deformed bulk Ta [3]. Twinning has been observed as a result of the indentation of thin films, but this has now been shown to be due to the formation of the fcc phase rather than twinning in the bcc phase [4].

In the present study we examine dislocations, in Ta, produced in different ways. First, thin films of Ta were deformed using nanoindentation. This method provides the model system; the thickness of the material can be varied and the films can be readily prepared for examination in the TEM. Then, different deformation processes were applied to bulk materials: indentation of pristine $\mathrm{Ta}$, dynamical deformation of pristine Ta, and indentation of material already dynamically tested. These methods provide the "real material" for comparison to the model system; the latter is particularly interesting since the material is presumably already heavily deformed so that the pre-existing defects strongly influence the mechanical deformation.

Figure 1 shows a low-magnification bright-field image of an indent in a thin Ta film on a Si substrate. The contrast in the image indicates that the film is polycrystalline with a somewhat columnar grain structure; this is a commonly observed microstructure in thin Ta films. The inset diffraction pattern identifies the bcc phase. Figure 2 is a high-resolution image from the indicated area in Fig. 1, where the strain is expected to be the highest. Although much of the image is dominated by $\{110\}$ fringes from the bcc phase, the inset FFT from the indicated region of interest shows spots corresponding to the $\{111\}$ planes of the fcc phase as reported in [2] and [3]. Figure 3 is an EBSD map of a dynamically deformed, indented Ta sample; the map was used to inform the location of FIB lift-outs and the TEM results will be presented. [6]

\section{References:}

[1] Bublik AI, Pines BIA (1952) Phase transition during the change of thickness in thin metal films. Dokl Akad Nauk USSR 87: 215-217

[2] Janish MT, Mook WM, Carter CB (2014) Nucleation of fcc Ta when heating thin films. Scripta Mater 96: 21-24

[3] Janish MT et al (2015) Observations of fcc and hcp Tantalum. J Mater Sci 50: 3706-3715 
[4] Wang JW, et al (2015) In situ atomic-scale observation of twinning-dominated deformation in nanoscale body-centred cubic tungsten. Nature Mater 14: 594-600

[5] Vachhani SJ et al (2015) Microstructure and local mechanical property evolution during high strain-rate deformation of tantalum. EPJ Web of Conferences 94: 02023-02021-02026

[6] MTJ would like to acknowledge a GAANN fellowship from the Department of Education. This work was partly performed at Sandia National Laboratory at the Center for Integrated Nanotechnologies, which is a DOE-BES-supported National User Facility. Sandia is a multiprogram laboratory operated by Sandia Corporation, a Lockheed Martin Company, for the US Department of Energy under contract DEAC04-94AL85000.

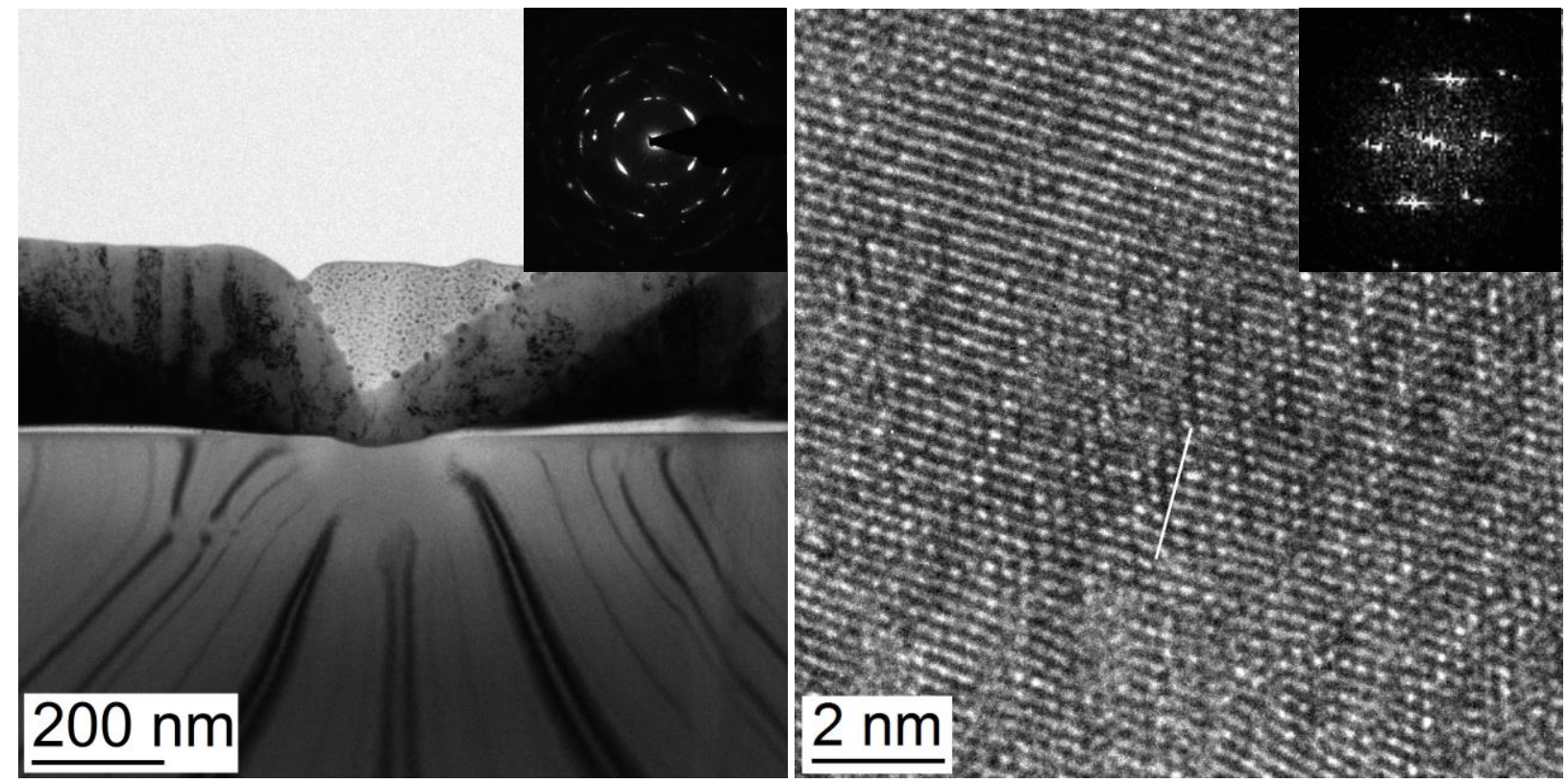

Fig 1. (left above) An example of an indent into a thin film of Ta on Si. The inset diffraction pattern clearly identifies the bcc phase. Fig. 2 (right above) High resolution image showing the polycrystalline nature of the material under an indent. There are clearly two sets of spots in the FFT; the angles between them correspond to both the $\{110\}$ planes in bcc Ta and the $\{111\}$ planes in fcc Ta.

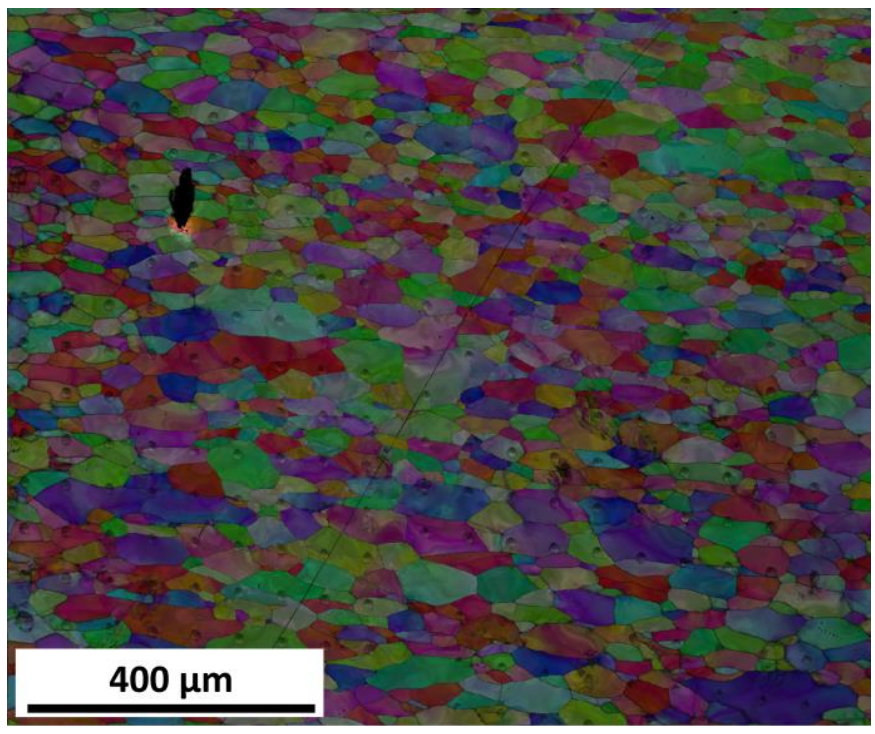

Fig 3. EBSD with superimposed quality-fit map with an array of indentations on polycrystalline Ta. The spacing of the indents ensures no overlap. TEM specimens can be FIBbed from inside grains, on grain boundaries or at indents. 